\title{
Rare, Serious, and Comprehensively Described Suspected Adverse Drug Reactions Reported by Surveyed Healthcare Professionals in Uganda
}

\author{
Ronald Kiguba $^{1}$, Charles Karamagi ${ }^{2}$, Paul Waako ${ }^{1}$, Helen B. Ndagije ${ }^{3}$, Sheila M. Bird ${ }^{4}$ \\ 1 Department of Pharmacology and Therapeutics, Makerere University College of Health Sciences, \\ Kampala, Uganda, 2 Clinical Epidemiology Unit, Makerere University College of Health Sciences, Kampala, \\ Uganda, 3 National Pharmacovigilance Centre, National Drug Authority, Kampala, Uganda, 4 Medical \\ Research Council Biostatistics Unit, Cambridge, United Kingdom \\ * kiguba@gmail.com
}

\section{G openaccess}

Citation: Kiguba R, Karamagi C, Waako P, Ndagije HB, Bird SM (2015) Rare, Serious, and Comprehensively Described Suspected Adverse Drug Reactions Reported by Surveyed Healthcare Professionals in Uganda. PLoS ONE 10(4): e0123974. doi:10.1371/journal.pone.0123974

Academic Editor: Antje Timmer, Carl von Ossietzky University of Oldenburg, GERMANY

Received: October 6, 2014

Accepted: February 24, 2015

Published: April 23, 2015

Copyright: @ 2015 Kiguba et al. This is an open access article distributed under the terms of the Creative Commons Attribution License, which permits unrestricted use, distribution, and reproduction in any medium, provided the original author and source are credited.

Data Availability Statement: All relevant data are within the paper and its Supporting Information files.

Funding: This work was supported by Training Health Researchers into Vocational Excellence (THRiVE) in East Africa, grant number 087540, funded by the Wellcome Trust to RK; grant number 5R24TW008886 supported by OGAC, National Institutes of Health $(\mathrm{NIH})$ and (Health Resources and Services Administration (HRSA) to RK; and an African Doctoral Dissertation Research Fellowship (ADDRF) award 2013-2015 ADF 006 offered by the African Population and Health Research Centre

\section{Abstract}

\section{Background}

Lack of adequate detail compromises analysis of reported suspected adverse drug reactions (ADRs). We investigated how comprehensively Ugandan healthcare professionals (HCPs) described their most recent previous-month suspected ADR, and determined the characteristics of HCPs who provided comprehensive ADR descriptions. We also identified rare, serious, and unanticipated suspected ADR descriptions with medication safetyalerting potential.

\section{Methods}

During 2012/13, this survey was conducted in purposively selected Ugandan health facilities (public/private) including the national referral and six regional referral hospitals representative of all regions. District hospitals, health centres II to IV, and private health facilities in the catchment areas of the regional referral hospitals were conveniently selected. Healthcare professionals involved in prescribing, transcribing, dispensing, and administration of medications were approached and invited to self-complete a questionnaire on ADR reporting. Two-thirds of issued questionnaires $(1,345 / 2,000)$ were returned.

\section{Results}

Ninety per cent (241/268) of HCPs who suspected ADRs in the previous month provided information on five higher-level descriptors as follows: body site (206), drug class (203), route of administration (127), patient age (133), and ADR severity (128). Comprehensiveness (explicit provision of at least four higher-level descriptors) was achieved by at least two-fifths (46\%, 124/268) of HCPs. Received descriptions were more likely to be comprehensive from HCPs in private health facilities, regions other than central, and those not involved in 
(APHRC) in partnership with the International Development Research Centre (IDRC) to RK. In UK, SMB is funded by Medical Research Council programme number MC_U105260794. The work here reported is solely the responsibility of the authors and does not necessarily represent the official views of the supporting offices. The funders had no role in study design, data collection and analysis, decision to publish, or preparation of the manuscript.

Competing Interests: SMB holds GSK shares. This does not alter the authors' adherence to PLOS ONE policies on sharing data and materials. The authors declare that they otherwise have no competing interests. teaching medical students. Overall, 106 serious and 51 rare previous-month suspected ADRs were described. The commonest serious and rare ADR was Stevens-Johnson syndrome (SJS); mostly associated with oral nevirapine or cotrimoxazole, but haemoptysis after diclofenac analgesia and paralysis after quinine injection were also described.

\section{Conclusion}

Surveyed Ugandan HCPs who had suspected at least one ADR in the previous month competently provided comprehensive ADR descriptions: more, indeed, than are received per annum nationally. Properly analyzed, and with local feed-back, voluntary ADR reports by HCPs could be an essential alerting tool for identifying rare and serious suspected ADRs in Uganda.

\section{Background}

Adverse drug reactions (ADRs) contribute to the global burden of ill-health[1-3]. Efficient ADR detection and reporting systems in sub-Saharan Africa (SSA) could promote more effective management of medicines and foster improvements in the quality of healthcare[4]. The detection or suspicion of ADRs by healthcare professionals (HCPs) is a first step in ADR reporting[5]. The ability of HCPs to suspect or recognize ADRs and ably to differentiate them from patients' illnesses and/or to attribute them across co-administered medications are, however, major challenges[6]. Ugandan HCPs involved in medical research and non-nurses were more likely to have suspected an ADR in the previous month, but previous-month receipt of a patient ADR complaint was the dominant factor in triggering ADR suspicion[5].

The spontaneous suspected ADR reporting system in Uganda currently requires completion, by HCPs, of a paper-based ADR report form, see S1 Appendix for details. Only a small proportion (13\%) of previous-month suspected ADRs that surveyed Ugandan HCPs detected in routine clinical practice were reported[5], consistent with ADR under-reporting elsewhere [7]. National statistics for voluntary ADR reporting to the Ugandan National Pharmacovigilance Centre (NPC) indicate that only $0.44 \%$ of HCPs report suspected ADRs annually[5].

Lack of adequate detail in the majority ( $80 \%)$ of suspected ADR reports submitted to the $\mathrm{NPC}[8]$ is an additional challenge. 'Completeness', as defined by Uppsala Monitoring Centre (UMC) [9], is a country-level quantitative measure of how complete is the information on 12 key details, see S2 Appendix, in Individual Case Safety Reports (ICSRs) that UMC receives from NPCs. We defined a HCP-level comprehensive ADR description as having at least four of the following five higher-level descriptors: body site, drug class, route of administration, patient's age, and ADR severity. Since we were testing the ability of HCPs to provide focal "free text" ADR descriptions, our target was to identify descriptions with, at most, one missing higher-level descriptor. Except for patient age, none of the other four basic descriptors is explicitly an ICSR key detail. Our higher-level descriptors could be the starting point for the completeness evaluation process by which NPCs elicit UMC's 12 key details, which include the information ideally needed to assess the causality of suspected ADRs: temporal relationship to drug intake, response to drug withdrawal, event or laboratory test abnormality, presence/absence of disease process or other drugs that can explain the ADR, and re-challenge if possible [10]. The large proportion of reports with inadequate detail, as submitted to the NPC, poses a challenge for signal detection and causality assessment of suspected ADRs: underestimation of 
the true risks of patients' medications is the result[11]. This study is focused on basic ADR description; we do not address ADR causality.

Ugandan HCPs are encouraged to submit comprehensive reports on suspected ADRs internally within the healthcare system and, ultimately, these reports ought to reach the NPC. Such submissions promote informed risk-benefit evaluation of medications and improve overall patient safety. We investigated how comprehensively Ugandan HCPs described their most recent previous-month suspected ADR, and determined the characteristics of HCPs who provided comprehensive ADR descriptions. We also identified rare, serious, and unanticipated suspected ADR descriptions with medication safety alerting potential.

\section{Methods}

\section{Study design and sampling procedure}

From 25 May 2012 to 28 February 2013, we conducted a cross-sectional study in Uganda in purposively selected, geographically diverse public and private health facilities. Details of the survey-design, anonymous self-completion of survey questionnaire, and sampling procedures have been described elsewhere[5]. Briefly, health facilities were selected to include the national referral hospital, and six regional referral hospitals which were selected to be representative of the Central, Eastern, Northern, Southern, and Western regions of Uganda. For practical purposes, district hospitals, health centres II to IV, and private health facilities (both for-profit and not-for-profit) in the catchment areas of the regional referral hospitals were conveniently selected. Healthcare professionals involved in prescribing, transcribing, dispensing medication orders, and administration of medicines to patients in the selected health facilities were eligible. Pre-trained research assistants approached HCPs and invited them to complete a pretested self-administered questionnaire. Healthcare professionals who accepted to participate gave separately-held written informed consent[5]. The self-completed questionnaires were tracked using serial numbers and they did not elicit identifying information on individual $\mathrm{HCPs}[5]$. Two-thirds of the issued questionnaires were returned.

\section{Data collection and management}

The self-administered questionnaire was used to obtain demographic and professional information; and description of the most recent previous-month suspected ADR[5], see S3 Appendix. Descriptions of the most recent suspected ADR were rated on the presence/absence of five higher-level descriptors: body site, drug class, route of administration, patient's age, and ADR severity. Comprehensiveness of ADR description was defined by the presence of at least four descriptors.

We distinguished between a severe suspected ADR and a serious suspected ADR. A serious $\mathrm{ADR}$ is one that can result in death, is life-threatening, requires initial or prolonged hospitalization, or results in disability or incapacitation[12]. Severity of an ADR describes its intensity as: mild, moderate or severe[13]. Thus, a severe ADR (severity grade 3 ) is not synonymous with a serious ADR[13].

We defined rare ADRs as those which are reported to occur less often than in 10 per 10,000 individuals on specified medication $(\leq 0.1 \%)[14]$; and unanticipated ADRs as those which, to our knowledge, are not supported by existing literature.

When invited to describe their most recent previous-month suspected ADR, some HCPs provided descriptions for two to three such cases. We used simple random sampling to retain one ADR description from each such set for analysis, see S4 Appendix.

All data were entered into a databank using EpiData 3.1. 


\section{Analysis}

Free-text descriptions for all ADRs and how we coded them for the five higher-level descriptors are provided in the S5 Appendix. Severity of ADRs, as reported by HCPs, was not altered by the study investigators in subsequent analyses.

Two investigators (RK \& SMB) each independently assessed the comprehensiveness of the 241 ADR descriptions: disagreements were resolved by consensus, and the consensus judgement on the five higher-level descriptors is provided in the S5 Appendix.

Seriousness of ADRs was assessed by RK based on the definition by the WHO Uppsala Monitoring Centre (UMC) classification[12] while the assessment of rareness and unanticipated ADR was conducted by RK using the online British National Formulary as the key reference guide[15].

Questionnaire responses and higher-level ADR descriptors were summarized as frequencies and percentages. We used logistic regression to assess demographic and professional determinants of comprehensiveness in describing the most recent of past-month suspected ADRs. Regression coefficients were expressed as odds ratios (ORs) with 95\% confidence intervals and were obtained using Stata 12.0[16]. We accounted for missing data using the missing-assigned approach where low-frequency missing data were meaningfully assigned to an existing category.

Descriptions of serious (incapacitating or life-threatening) suspected ADRs were tabulated by drug class, see S6 Appendix; and for similar listing of severe (grade 3 intensity) suspected ADRs, see S7 Appendix. For ease of assimilation, rare suspected ADRs were grouped by whether or not SJS was explicitly mentioned; if not, rare ADRs were listed by the named drug.

\section{Ethical clearance}

The School of Medicine Research and Ethics Committee, Makerere University College of Health Sciences, and the Uganda National Council for Science and Technology provided ethical approval for the study.

\section{Results}

\section{Study population}

Overall return-rate for the survey was $67 \%(1,345 / 2,000)$ and the mean number of patients seen per day by HCPs was 41 [(SD = 46), median of 30 (interquartile range from 15 to 50 patients/day; $\mathrm{n}=1,226$ of 1,345$)]$. Only $21 \%(268 / 1,289)$ of HCPs reported that they had suspected an ADR in the previous month. The mean age of respondent HCPs who suspected an $\mathrm{ADR}$ in the previous 4 -weeks was 32.2 years $(\mathrm{SD}=8.5 ; \mathrm{n}=222$ of 268$)$. Twenty seven $(10 \%)$ of the $268 \mathrm{HCPs}$ who suspected an ADR in the previous month did not provide any ADR description. Of the 241 who did, 95 (39\%) were nurses, 77 (32\%) were doctors, 23 (10\%) were pharmacists/pharmacy technicians and 46 (19\%) were other cadres.

\section{Suspected ADRs}

Of the 268 HCPs who had suspected an ADR in the previous month, 241 (90\%) provided a free-text description of the most recently encountered previous-month suspected ADR with higher-level details given as follows: body-site [85\%, 206/241; skin only reactions were the modal group (43\%, 88/206)]; drug class [84\%, 203/241; with the top three single classes cited as follows: antibacterials (70), antiretrovirals (45), and antimalarials (28)]; route of administration [(explicitly cited: 53\%, 127/241), although, in addition, route could be inferred from the named-drug in a further 63 descriptions (hence, $79 \%$ ); with 50 of 190 being by injection]; 
patient's age [ $55 \%, 133 / 241$; mean age 28.3 years ( $S D=16.5$ ), median of 28 years and interquartile range from 20 to 36 years]; and ADR-severity [53\%, 128/241; with 46\% (59/128) of citations being in respect of severe reactions], see Table 1 and $S 4$ Appendix.

\section{Comprehensive survey-described suspected ADRs}

Comprehensiveness of ADR description was achieved by 46\% (124/268; 95\% CI: $40 \%$ to 52\%) of HCPs when only 85 would have been expected by independence of descriptors, see Table 2 .

The more comprehensive ADR descriptions were received from HCPs in private health facilities [vs. public; private not-for-profit ( $\mathrm{OR}=3.4,95 \% \mathrm{CI}$ : 1.48 to 7.82$) \&$ private for-profit $(\mathrm{OR}=2.6,95 \% \mathrm{CI}: 1.13-5.96)$ ], and regions other than Central Uganda [Eastern $(\mathrm{OR}=2.6$, 95\% CI: 1.20-5.73) \& combined Western-Northern-Southern (OR = 6.4, 95\% CI: 2.85-14.37)]. Comprehensive ADR description was less likely from HCPs who were involved in teaching medical students (OR $=0.5,95 \%$ CI: $0.23-1.00$ ) and was not significantly influenced by professional cadre (nurse versus non-nurse), life-time encountering of a fatal ADR, age of HCP, receipt of a previous-month patient ADR complaint, involvement in medical research, patient load, department, and knowing to whom to report ADRs, see Table 3.

\section{Serious suspected ADRs}

Serious suspected ADRs constituted 44\% (106/241) of the ADR descriptions; see S6 Appendix for list of the 106 serious suspected ADRs. The most frequent body sites of serious ADRs were skin only $(33 \%, 34 / 102)$, central nervous system (CNS) only (11\%, 11/102), and gastrointestinal tract (GI) only $(8 \%, 8 / 102)$, see Table 4 . The most frequent single drug classes of serious ADRs were antiretrovirals $(35 \%, 31 / 88)$, antibacterials $(15 \%, 13 / 88)$ and antimalarials $(14 \%, 12 / 88)$, see Table 4. Serious suspected ADRs accounted for only 24\% (13/53: 95\% CI: 13\%- 36\%) of antibacterial-only-linked ADR descriptions but for 82\% (31/38: 95\% CI: 70\%- 94\%) of antiretroviral-only-linked suspected ADR descriptions, and for 46\% (12/26: 95\% CI: 27\%- 65\%) of antimalarial-only-linked suspected ADRs [Chi $(2 \mathrm{df})=29.35$; $\mathrm{P}<0.001$ ]. Severity (grade or intensity) was not reported for half (53/106) of the serious (incapacitating or life-threatening) ADRs.

\section{Case reports of serious suspected ADRs}

A 28-year-old patient received cotrimoxazole tablets and developed black patches on the skin (reported by a 35-year-old female registered midwife in a public health centre IV, Tororo, Eastern Uganda); severe haemoptysis (coughing up of blood) in a patient after two days on oral diclofenac (reported by a 28-year-old male doctor in a private for-profit hospital in Kampala, Central Uganda); and, a 17-year-old receiving intramuscular quinine developed severe post injection paralysis (reported by a 60 -year-old female enrolled nurse in a private-not-for-profit hospital in Mbarara district, Southern Uganda), see S8 Appendix.

\section{Rare suspected ADRs}

Fifty one rare suspected ADRs (which occur in $\leq 0.1 \%$ of medication users) were reported, of which 65\% (33/51) were serious, see Tables 4 and 5. Among the rare ADR descriptions, Stevens-Johnson syndrome (SJS) was explicitly mentioned in 13 of 51 cases and implicated drugs were: antiretroviral therapy [five of 13 cases were nevirapine-linked, and one was linked to zidovudine-lamivudine post-exposure prophylaxis]; cotrimoxazole (septrin) [four]; ciprofloxacin [one]; carbamazepine [one]; and no drug mentioned [one]. 
Table 1. Survey-description of the most recent past-month suspected ADR provided by 241 out of 268 HCPs who suspected ADRs in the previous month, Uganda, 2013.

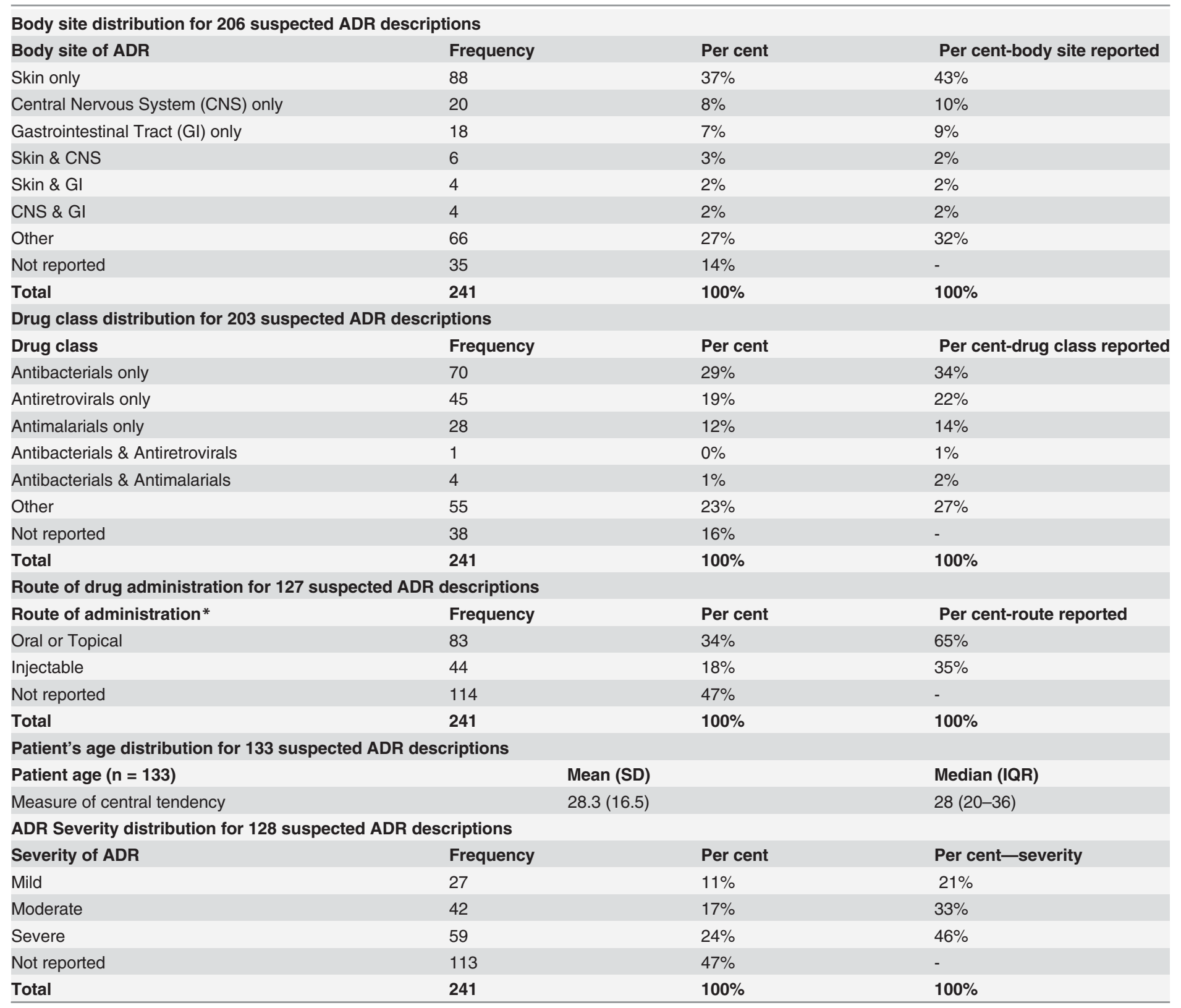

* Route of administration could be inferred for a further 63 ( 6 were by injection \& 59 were oral) ADR descriptions.

doi:10.1371/journal.pone.0123974.t001

Additional ADR descriptions involving skin ulcerations, blistering, sloughing/peeling, and black skin patches; burnt face and lips; sore mucous membranes or general body sores; and death were linked to cotrimoxazole [12 of 38 cases] and nevirapine [1 of 38 cases] administration. Severe urticaria, swelling of the face, hands, and injection site were associated with ciprofloxacin use [3 of 38 cases]. Death, miscarriage, skin blistering and body itching were associated with quinine use [8 of 38 cases]. Severe palpitations due to overdose of omeprazole [ 1 case]; haemoptysis (coughing up of blood) after oral administration of diclofenac [1 case]; 
Table 2. Comprehensiveness of ADR-description of the most recent past-month suspected ADR(s) as reported by 241 out of 268 HCPs who suspected ADRs in the previous month, Uganda, 2013.

\begin{tabular}{|c|c|c|c|c|c|c|c|}
\hline $\begin{array}{l}\text { HIGHER-LEVEL DETAILS in addition } \\
\text { to ADR }\end{array}$ & $\begin{array}{l}\text { Body } \\
\text { Site }\end{array}$ & $\begin{array}{l}\text { Drug } \\
\text { Class }\end{array}$ & Route & $\begin{array}{l}\text { Age of } \\
\text { patient }\end{array}$ & Severity & $\begin{array}{l}\text { Observed } \\
\text { number }\end{array}$ & $\begin{array}{l}\text { Expected by } \\
\text { independence }\end{array}$ \\
\hline \multirow[t]{2}{*}{ All 5 provided } & $\sqrt{ }$ & $\sqrt{ }$ & $\sqrt{ }$ & $\sqrt{ }$ & $\sqrt{ }$ & 54 & 17 \\
\hline & $206 / 268$ & $203 / 268$ & $\begin{array}{l}127 / \\
268\end{array}$ & $133 / 268$ & $\begin{array}{l}128 / \\
268\end{array}$ & & \\
\hline \multicolumn{8}{|l|}{ Combinations of 4} \\
\hline Combination 1 & $\sqrt{ }$ & $\sqrt{ }$ & $\sqrt{ }$ & $\sqrt{ }$ & & 22 & 19 \\
\hline Combination 2 & & $\sqrt{ }$ & $\sqrt{ }$ & $\sqrt{ }$ & $\sqrt{ }$ & 10 & 5 \\
\hline Combination 3 & $\sqrt{ }$ & $\sqrt{ }$ & & $\sqrt{ }$ & $\sqrt{ }$ & 19 & 20 \\
\hline Combination 4 & $\sqrt{ }$ & & $\sqrt{ }$ & $\sqrt{ }$ & $\sqrt{ }$ & 2 & 6 \\
\hline Combination 5 & $\sqrt{ }$ & $\sqrt{ }$ & $\sqrt{ }$ & & $\sqrt{ }$ & 17 & 18 \\
\hline \multicolumn{5}{|c|}{ TOTAL } & & 124 & 85 \\
\hline
\end{tabular}

doi:10.1371/journal.pone.0123974.t002

and mental confusion due to mefloquine [ 1 case] were also reported; for 11 others cases, see Table 5 .

\section{Unanticipated suspected ADRs}

Six unanticipated suspected ADRs (not backed by existing literature) were described: generalized body sores after oral coartem (artemether-lumefantrine); hypotension and sweating after intravenous diclofenac; reduced blood pressure and low pulse after chloramphenicol injection; severe burning sensation in the private parts immediately after intravenous hydrocortisone; irritability after intravenous quinine; and shaking and sweating for 10 minutes after receiving paracetamol, see S4 Appendix.

\section{Summary of suspected ADRs}

In summary, 106 serious, 51 rare, and six unanticipated past-month ADRs were described by 1,345 respondent $\mathrm{HCPs}$, who, on average, saw 41 patients daily $(\mathrm{SE}=1.3)$. Including overlaps between the patients seen, the reporting period accounts for over 1.5 million patient-days $\left(1,345^{*} 41^{*} 28=1,544,060\right.$ patient-days). If our respondents can be considered representative, and for future planning, we estimate that, per 4-weeks, 100 Ugandan HCPs may encounter eight (8) serious, four (4) rare, and fewer than one (0.5) unanticipated suspected ADRs.

\section{Discussion}

Ninety percent (241/268) of surveyed HCPs who suspected an ADR in the previous month described the most recent past-month suspected ADR they had encountered. Although the proportion of HCPs who provided these ADR description(s) was high, elsewhere we have reckoned[5] that only a small proportion (13\%) of previous-month suspected ADRs that our surveyed Ugandan HCPs detected in routine clinical practice was formally reported. Moreover, the number of ADR reports (241 reports from 1,345 HCPs) in our study was higher than the Ugandan National Pharmacovigilance Centre's annual mean number of ADR reports (207 reports from 46,566 HCPs) over the previous six and a half years[5]. Our study, therefore, demonstrated a high level of motivation to describe the most recent previous-month suspected ADRs amongst the surveyed HCPs; however, the impetus for voluntary reporting of suspected ADRs in routine clinical practice is still very low [5]. 
Table 3. Personal and professional factors associated with comprehensive ADR description of most recent past-month suspected ADR for 241 healthcare professionals who suspected ADR(s) in the previous month and provided a description, Uganda, 2013.

\begin{tabular}{|c|c|c|c|c|c|c|c|c|}
\hline \multirow[t]{2}{*}{ Factor } & \multicolumn{2}{|c|}{$\begin{array}{l}\text { Comprehensive } \\
\text { ADR Description }\end{array}$} & \multicolumn{3}{|c|}{ Crude Analysis } & \multicolumn{3}{|c|}{ Adjusted Analysis } \\
\hline & Yes (\%) & No (\%) & OR & $95 \% \mathrm{Cl}$ & P-value & OR & $95 \% \mathrm{Cl}$ & P-value \\
\hline \multicolumn{9}{|l|}{ Level of Health Facility } \\
\hline Other & $37(56)$ & $29(44)$ & 1.0 & & & 1.0 & & \\
\hline Hospital & $87(50)$ & $88(50)$ & 0.8 & $0.44-1.37$ & 0.380 & 2.0 & $0.86-4.47$ & 0.110 \\
\hline \multicolumn{9}{|l|}{ Type of Health Facility } \\
\hline Public & $49(41)$ & $71(59)$ & 1.0 & & & 1.0 & & \\
\hline Private Not-For-Profit & $31(67)$ & $15(33)$ & 3.0 & $1.46-6.13$ & 0.003 & 3.4 & $1.48-7.82$ & 0.004 \\
\hline Private For-Profit & $44(59)$ & $31(41)$ & 2.1 & $1.14-3.70$ & 0.016 & 2.6 & $1.13-5.96$ & 0.024 \\
\hline \multicolumn{9}{|l|}{ Region of the country } \\
\hline Central & $49(37)$ & $82(63)$ & 1.0 & & & 1.0 & & \\
\hline Eastern & $34(62)$ & $21(38)$ & 2.7 & $1.42-5.18$ & 0.003 & 2.6 & $1.20-5.73$ & 0.015 \\
\hline Other & $41(75)$ & $14(25)$ & 4.9 & $2.43-9.89$ & $<0.001$ & 6.4 & $2.85-14.37$ & $<0.001$ \\
\hline \multicolumn{9}{|l|}{ Professional Cadre } \\
\hline Nurse & $54(57)$ & $41(43)$ & 1.0 & & & 1.0 & & \\
\hline Non-nurse & $70(48)$ & $76(52)$ & 0.7 & $0.42-1.18$ & 0.178 & 1.1 & $0.54-2.32$ & 0.769 \\
\hline \multicolumn{9}{|l|}{ Age } \\
\hline Less than 30 years & $53(50)$ & $54(50)$ & 1.0 & & & 1.0 & & \\
\hline Aged 30 years or older & $71(53)$ & $63(47)$ & 1.1 & $0.69-1.91$ & 0.594 & 1.7 & $0.92-3.09$ & 0.090 \\
\hline \multicolumn{9}{|l|}{ Patient Load } \\
\hline Greater than 30/day & $59(50)$ & $58(50)$ & 1.0 & & & 1.0 & & \\
\hline At most 30/day & $65(52)$ & $59(48)$ & 1.1 & $0.65-1.80$ & 0.757 & 1.4 & $0.78-2.66$ & 0.241 \\
\hline \multicolumn{9}{|l|}{ Department } \\
\hline Medicine & $70(52)$ & $64(48)$ & 1.0 & & & 1.0 & & \\
\hline Surgery & $4(31)$ & $9(69)$ & 0.4 & $0.12-1.38$ & 0.150 & 0.4 & $0.09-1.47$ & 0.156 \\
\hline Paediatrics, OBS \& Gyn & $16(43)$ & $21(57)$ & 0.7 & $0.33-1.45$ & 0.334 & 0.7 & $0.29-1.64$ & 0.404 \\
\hline Other & $34(60)$ & $23(40)$ & 1.4 & $0.72-2.53$ & 0.347 & 1.0 & $0.48-2.04$ & 0.973 \\
\hline \multicolumn{9}{|l|}{ Teaching medical students } \\
\hline No & $101(57)$ & $75(43)$ & 1.0 & & & 1.0 & & \\
\hline Yes & $23(35)$ & $42(65)$ & 0.4 & $0.23-0.73$ & 0.003 & 0.5 & $0.23-1.00$ & 0.050 \\
\hline \multicolumn{9}{|l|}{ Ever encountered fatal ADR } \\
\hline No & $98(55)$ & $79(45)$ & 1.0 & & & 1.0 & & \\
\hline Yes & $26(41)$ & $38(59)$ & 0.6 & $0.31-0.99$ & 0.044 & 0.7 & $0.35-1.42$ & 0.327 \\
\hline \multicolumn{9}{|c|}{ Involved in medical research } \\
\hline No & $75(51)$ & $71(49)$ & 1.0 & & & 1.0 & & \\
\hline Yes & $49(52)$ & $46(48)$ & 1.0 & $0.60-1.69$ & 0.975 & 1.8 & $0.96-3.43$ & 0.068 \\
\hline \multicolumn{9}{|l|}{ Gender } \\
\hline Male & $57(47)$ & $64(53)$ & 1.0 & & & 1.0 & & \\
\hline Female & $67(56)$ & $53(44)$ & 1.4 & $0.85-2.36$ & 0.176 & 1.4 & $0.70-2.82$ & 0.334 \\
\hline \multicolumn{9}{|c|}{ Suggestions for improved ADR reporting* } \\
\hline No & $21(49)$ & $22(51)$ & 1.0 & & & & & \\
\hline Yes & $103(52)$ & $95(48)$ & 1.1 & $0.59-2.20$ & 0.705 & & & \\
\hline \multicolumn{9}{|l|}{ Knows to whom to report* } \\
\hline No & $51(45)$ & $63(55)$ & 1.0 & & & & & \\
\hline Yes & $73(58)$ & $54(42)$ & 1.7 & $1.00-2.78$ & 0.049 & & & \\
\hline Patient ADR complaint* & & & & & & & & \\
\hline
\end{tabular}


Table 3. (Continued)

\begin{tabular}{|c|c|c|c|c|c|c|c|c|}
\hline \multirow[t]{2}{*}{ Factor } & \multicolumn{2}{|c|}{$\begin{array}{l}\text { Comprehensive } \\
\text { ADR Description }\end{array}$} & \multicolumn{3}{|c|}{ Crude Analysis } & \multicolumn{3}{|c|}{ Adjusted Analysis } \\
\hline & Yes (\%) & No (\%) & OR & $95 \% \mathrm{Cl}$ & P-value & OR & $95 \% \mathrm{Cl}$ & P-value \\
\hline No & $35(55)$ & $29(45)$ & 1.0 & & & & & \\
\hline Yes & $89(50)$ & $88(50)$ & 0.8 & $0.47-1.49$ & 0.546 & & & \\
\hline
\end{tabular}

doi:10.1371/journal.pone.0123974.t003

Half of the ADR descriptions provided (124/241) were comprehensive. The extent of comprehensive ADR description is laudable given the fact that we elicited information on the most recent previous-month suspected ADR by recall and without prior sensitization of the HCPs about this survey. Half of the reports explicitly cited route of administration but route could nonetheless be inferred from the named drug in a further $26 \%$ (63/241) of descriptions. To eliminate errors in analysis of reported suspected ADRs, HCPs ought always to report drug route clearly.

Completeness and relevance are VigiBase database measures coined by the Uppsala Monitoring Centre (UMC) to assess the amount and quality of detail in Individual Case Safety Reports (ICSRs) received from National Pharmacovigilance Centres[9]. Completeness is quantitative and it measures to what level an ICSR is complete after NPC has liaised with the HCP-reporter, see S2 Appendix, while relevance is qualitative and can be used to assess causal relationships between a drug and an ADR[9]. The relevance algorithm, however, is still being developed[9]. Our findings could enhance the process of developing algorithms used to assess the level of detail and quality of information in ADR reports, such as need for explicit route of administration. It is the responsibility of individual countries to improve the quality of ADR reports that they receive from HCPs.

Comprehensive "free text" suspected ADR description provides additional detailed qualitative information that could enhance "completeness" and "relevance" assessment of ICSRs by NPCs, and generates stronger medication safety signals which, in turn, might promote proper characterization of reported suspected ADRs. More certainty about the nature of suspected ADRs and efficiency in taking appropriate measures to prevent these medication safety threats to patients is the result. Determining the characteristics of HCPs who are less likely to provide comprehensive ADR reports can help to identify HCPs to whom interventions aimed at improving the comprehensiveness (and ultimately overall quality) of ADR reporting can be targeted while at the same time utilizing the skills of the more comprehensive ADR reporters to strengthen/support improvements in the overall quality of suspected ADR reporting in Uganda.

Published literature which gives an in-depth account of the level of detail in ADR descriptions reported by HCPs in sub-Saharan Africa (SSA) was lacking. This study, therefore, provided empirical data on HCP-described ADRs in diverse health facilities in a Ugandan setting. The aim was to stimulate further research and intellectual discourse on comprehensiveness of ADR reporting in SSA in order to inspire similar, periodic but prospectively designed studies, to understand and improve the overall quality of ADR-reporting by HCPs in limited-resource settings where the use of technology-aided electronic ADR signal detection in daily clinical practice is not yet widely implemented. We assessed five higher-level descriptors provided in free-text recalled descriptions, but acknowledge that suspected ADR reports ought also to have relevant information to facilitate ADR causality assessment[10] and risk-benefit evaluation of 
Table 4. Seriousness of the most recent past-month suspected ADRs described by 241 out of 268 HCPs who suspected ADRs in the previous month, Uganda, 2013.

\begin{tabular}{|c|c|c|c|c|}
\hline \multicolumn{5}{|c|}{ Serious ADR distribution for 241 suspected ADR descriptions ( $n, \%)$} \\
\hline Serious ADR & \multicolumn{2}{|c|}{ Body Site Specified } & Drug Class Specified & Frequency \\
\hline Yes & \multicolumn{2}{|c|}{$102(50)$} & $88(43)$ & $106(44)$ \\
\hline No & \multicolumn{2}{|c|}{$93(45)$} & $84(41)$ & $97(40)$ \\
\hline Unassessable & \multicolumn{2}{|c|}{$11(5)$} & $31(15)$ & $38(16)$ \\
\hline Total & \multicolumn{2}{|c|}{$206(100)$} & $203(100)$ & $241(100)$ \\
\hline \multicolumn{5}{|c|}{ Serious ADR by Body Site for 206 suspected ADR descriptions } \\
\hline \multirow[t]{2}{*}{ Body Site } & & \multicolumn{3}{|c|}{ Serious ADR (n, \%) } \\
\hline & Yes & No & Unassessable & Total \\
\hline Skin only* & $34(33)$ & $47(51)$ & $7(64)$ & $88(43)$ \\
\hline CNS only* & $11(11)$ & $9(10)$ & $0(0)$ & $20(10)$ \\
\hline GI only* & $8(8)$ & $9(10)$ & $1(9)$ & $18(9)$ \\
\hline Skin \& CNS & $3(3)$ & $3(3)$ & $0(0)$ & $6(3)$ \\
\hline Skin \& Gl & $1(1)$ & $3(3)$ & $0(0)$ & $4(2)$ \\
\hline CNS \& GI & $2(2)$ & $2(2)$ & $0(0)$ & $4(2)$ \\
\hline Other & $43(42)$ & $20(21)$ & $3(27)$ & $66(31)$ \\
\hline Total & $102(100)$ & $93(100)$ & $11(100)$ & $206(100)$ \\
\hline \multicolumn{5}{|c|}{ Serious ADR by Drug Class for 203 suspected ADR descriptions } \\
\hline \multirow[t]{2}{*}{ Drug Class } & \multicolumn{4}{|c|}{ Serious ADR (n, \%) } \\
\hline & Yes & No & Unassessable & Total \\
\hline Antibacterials only* & $13(15)$ & $40(48)$ & $17(55)$ & $70(34)$ \\
\hline Antiretrovirals only* & $31(35)$ & $7(8)$ & $7(23)$ & $45(22)$ \\
\hline Antimalarials only* & $12(14)$ & $14(17)$ & $2(6)$ & $28(14)$ \\
\hline Antibacterial \& Antiretroviral & $0(0)$ & $0(0)$ & $1(3)$ & $1(1)$ \\
\hline Antibacterials \& Antimalarials & $4(4)$ & $0(0)$ & $0(0)$ & $4(2)$ \\
\hline Other & $28(32)$ & $23(27)$ & $4(13)$ & $55(27)$ \\
\hline Totals & $88(100)$ & $84(100)$ & $31(100)$ & $203(100)$ \\
\hline \multirow[t]{2}{*}{ Rare ADR } & \multicolumn{4}{|c|}{ Serious ADR (n, \%) } \\
\hline & Yes & No & Unassessable & Total \\
\hline Yes & $33(31)$ & $18(19)$ & $0(0)$ & $51(21)$ \\
\hline No & $46(43)$ & $57(59)$ & $6(16)$ & $109(45)$ \\
\hline Unknown & $27(25)$ & $22(22)$ & $32(84)$ & $81(34)$ \\
\hline Total & $106(100)$ & $97(100)$ & $38(100)$ & $241(100)$ \\
\hline
\end{tabular}

Chi-square $(2 \mathrm{df})=6.42, \mathrm{P}<0.025$ (if the "unassessable" column is excluded)

*Proportions of serious ADRs (across rows), and their 95\% confidence intervals (95\% Cl) for a) body site: skin only, $39 \%$ (95\% Cl: $29 \%-49 \%)$; CNS only, 55\% (95\% Cl: 33\%- 77\%); Gl only, 44\% (95\% Cl: 21\%- 67\%); \& other, $\mathbf{4 2} \%$ (95\% Cl: 30\%- 54\%); and for, b) drug class: antibacterials only, 24\% (95\% Cl: 13\%- 36\%); antimalarials only, 46\% (95\% Cl: $27 \%-65 \%)$; antiretrovirals only, $82 \%$ (95\% Cl: $70 \%-94 \%)$; \& other, $58 \%$ (95\% Cl: $45 \%-71 \%)$

doi:10.1371/journal.pone.0123974.t004

medicines used in daily clinical practice[17]. For this reason, we recommend that a prospective version of our survey-approach to recording suspect ADRs be put in place by hospitals: for example, in two randomly selected months every year. Prospective recording during the survey months not only allows HCPs' multiple reports of the same suspected ADR to be identified but also allows contemporary consideration of causality and of the ward's medication-frequency for implicated drugs.

Working at private health facilities was associated with more comprehensive ADR descriptions. Thus, whereas past-year ADR reporting was lower in private-for-profit health facilities 


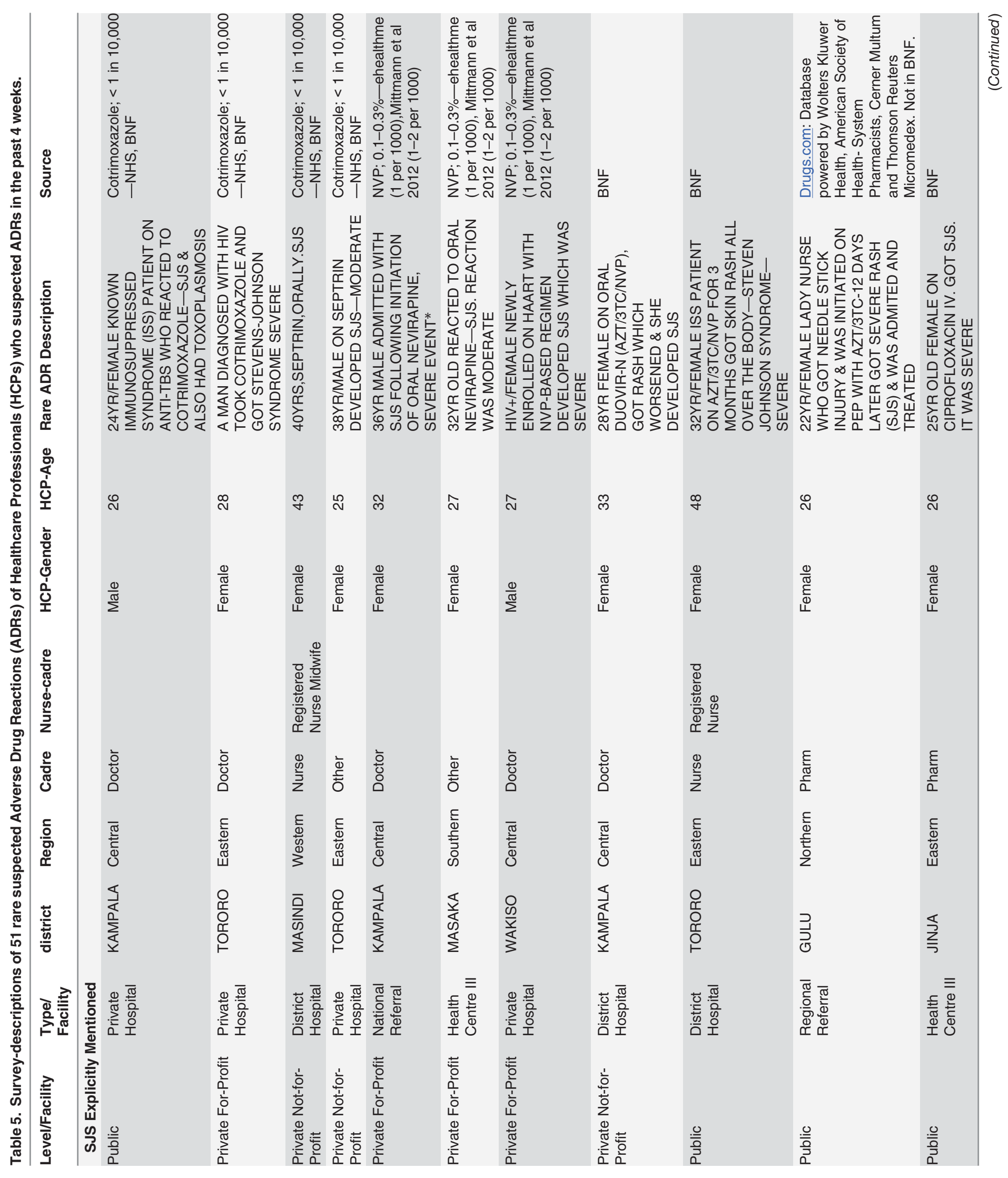




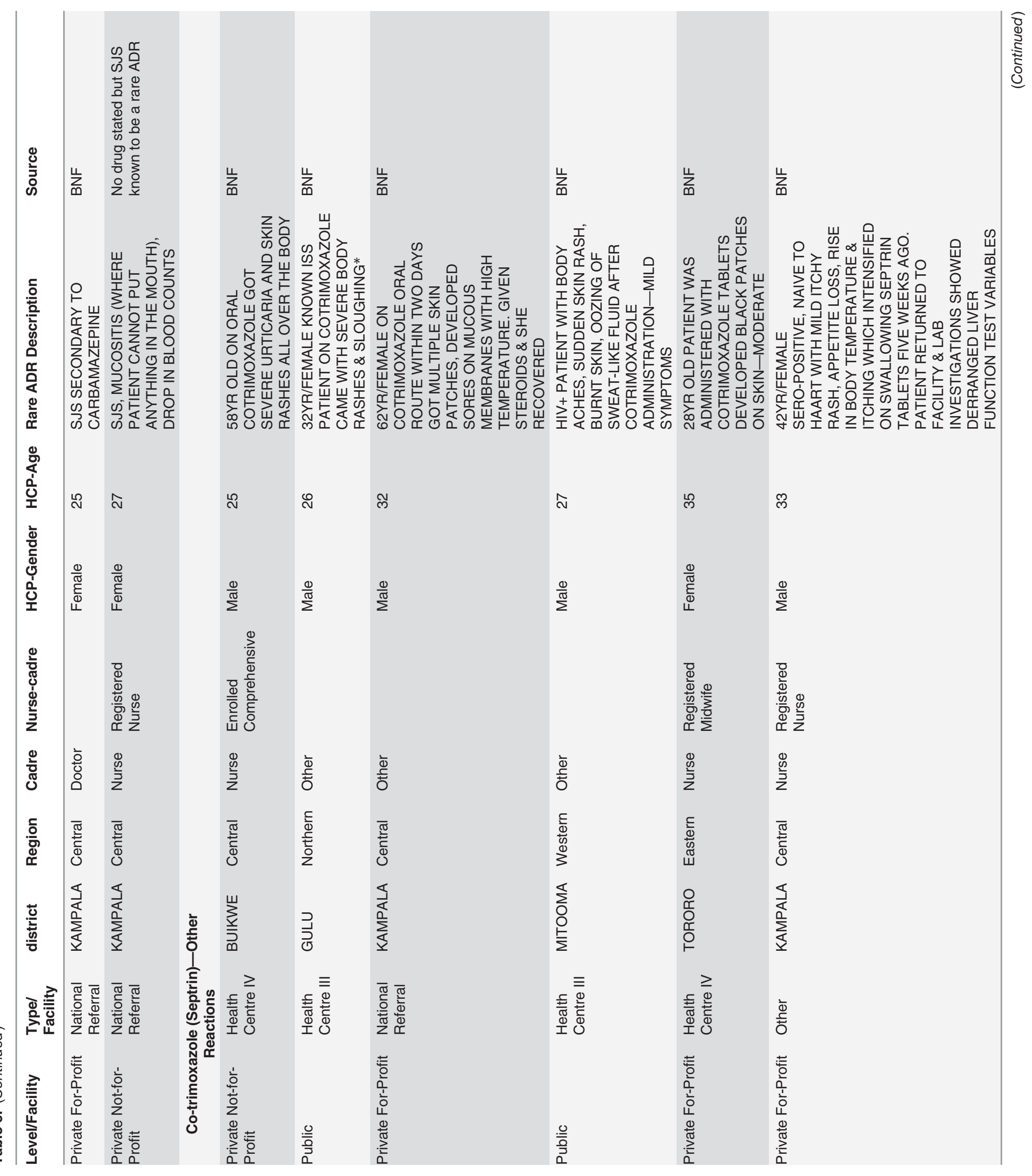




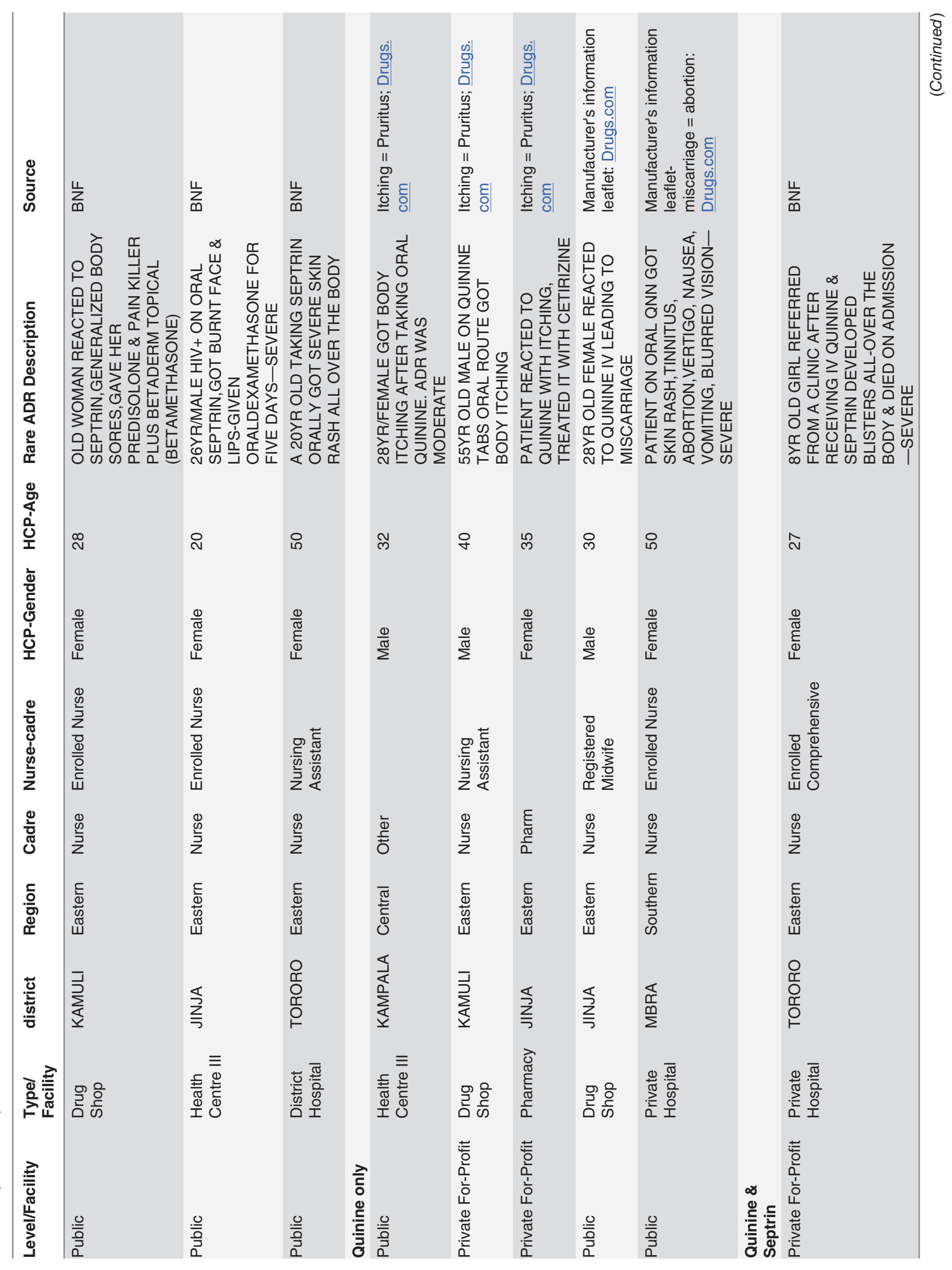




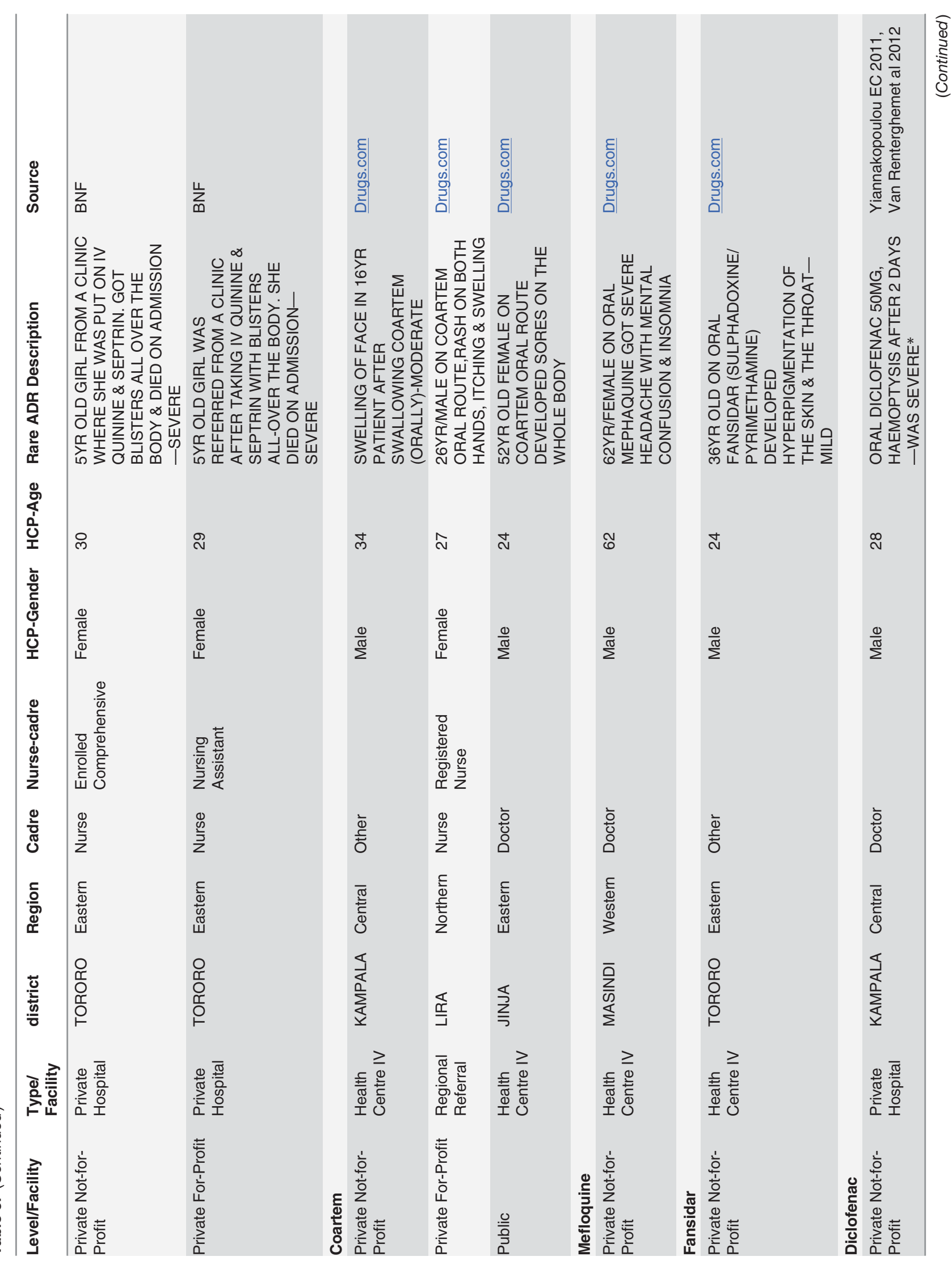




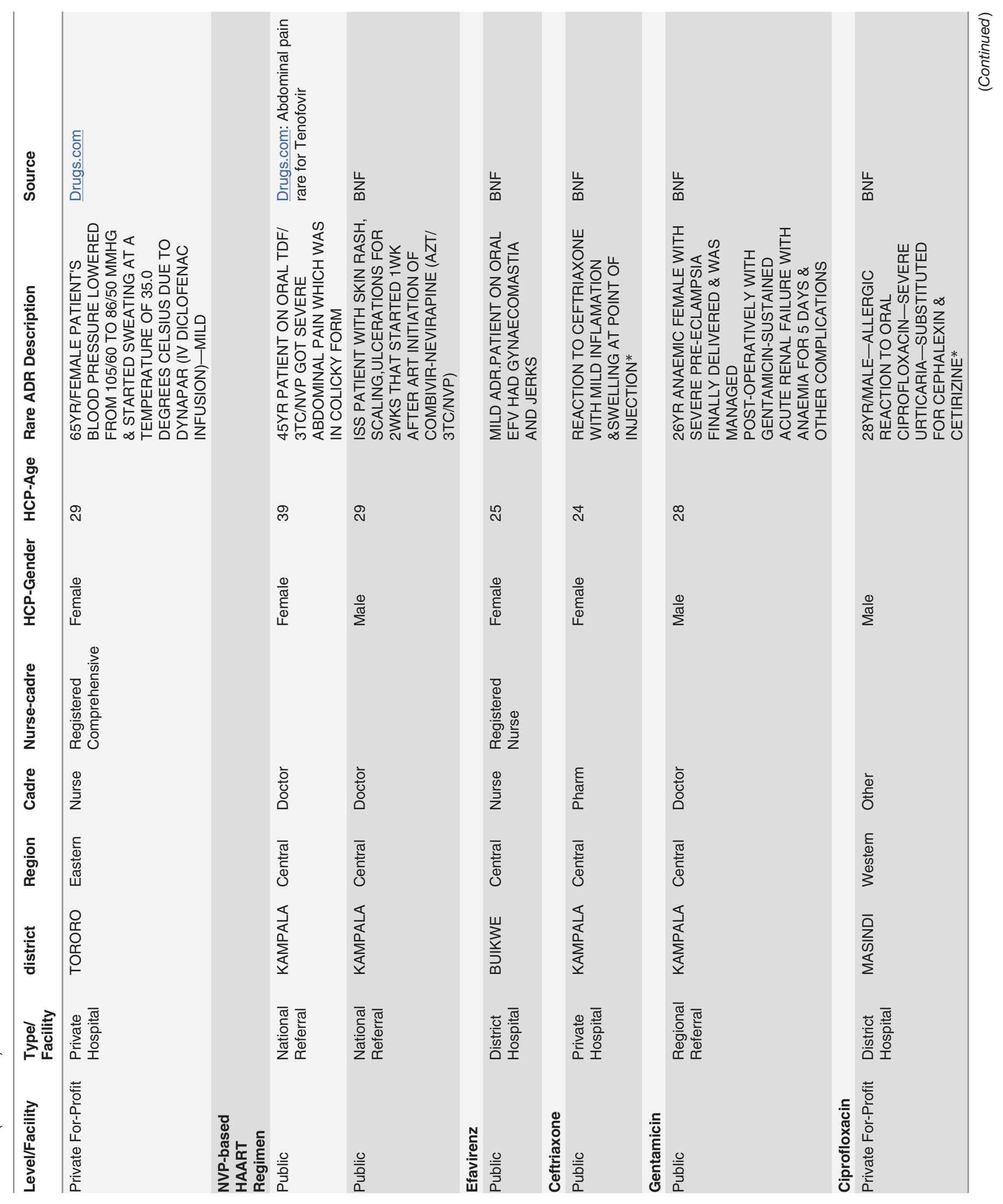




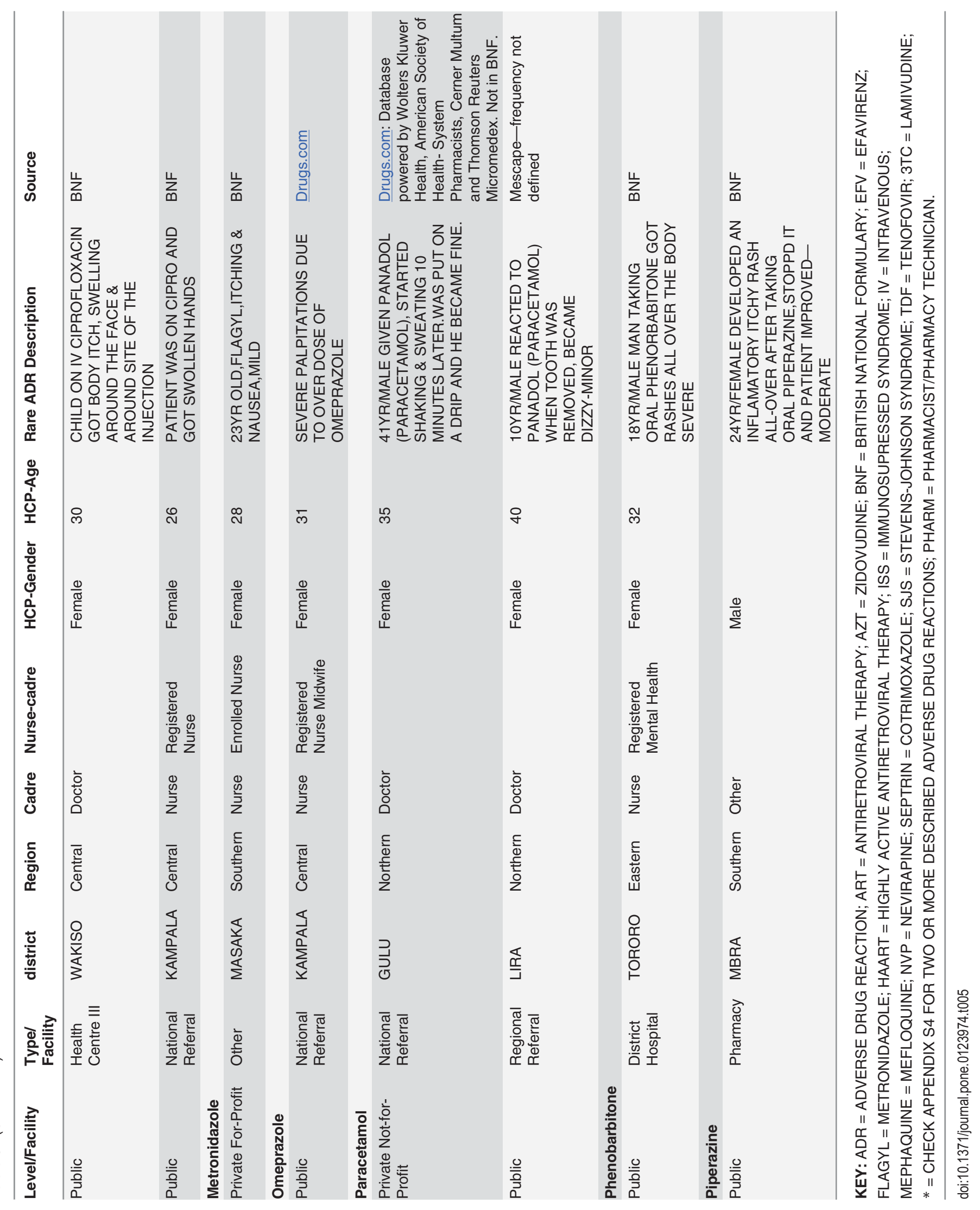


as compared with the public health sector[5], survey ADR descriptions from HCPs in private settings were three times more likely to be comprehensive. Any future interventions aimed at raising the ADR reporting rate by $\mathrm{HCPs}$ in both private and public health facilities should take into account both stimulation of, and the quality of, submitted ADR reports.

Healthcare professionals in regions other than in central Uganda had a higher likelihood to report comprehensive ADR descriptions. This finding might reflect the intensive NPC-led pharmacovigilance training focused on HCPs in regional referral hospitals when regional pharmacovigilance centres were first established in Uganda.

Healthcare professionals who were involved in the teaching of medical students were less likely to provide comprehensive ADR descriptions and yet it is these HCPs who bear the responsibility of introducing, to the medical students, the basic principles of pharmacovigilance during the formative years of the students' medical training. Targeted interventions to improve the comprehensiveness of ADR reports received from HCPs in teaching hospitals as well as lower level health facilities are essential to the strengthening of grassroots pharmacovigilance in Uganda. To stimulate more comprehensive ADR reporting, tables of serious and rare ADRs, such as those reported in this paper, should be provided both locally and nationally as feedback to HCPs. These tables group the received ADR reports meaningfully and indicate the rarity of the described ADR, where available, according to conventional references.

Lifetime encountering of a fatal ADR was not related to comprehensive ADR reporting. Thus, whereas occurrence of a fatal ADR might stimulate ADR reporting[5], it may not be an important factor in determining the comprehensiveness of reporting on suspected ADRs. Professional cadre, age of HCP, receipt of a previous-month patient ADR complaint, involvement in medical research, patient load, department, and knowing to whom to report ADRs were not statistically significantly associated with the reporting of comprehensive ADR descriptions among the surveyed HCPs.

The comparative advantage of post-marketing pharmacovigilance over randomized control trials (RCTs) is the ability to detect rare, serious, unanticipated, and/or delayed suspected ADRs that occur after a new drug product has been licensed for use on the open market. Moreover, RCTs are conducted in fewer subjects and in an experimental environment that may not adequately reflect the conditions of the "real word" healthcare setting $[18,19]$. Spontaneous and voluntary ADR reporting systems provide an inexpensive post-marketing pharmacovigilance system that can be used to identify and prevent ADRs (e.g. rare and serious ones) and other drug-related problems.

Severity (grade or intensity) was not reported for half of the serious (incapacitating or lifethreatening) ADRs suggesting that Ugandan HCPs rate seriousness higher than severity or that they do not take reporting of severity to be as important.

Miscarriage, post-injection paralysis, and child deaths were examples of serious ADRs associated with quinine use. Post-injection paralysis has previously been found to be associated with the bad clinical practice of inappropriate injection of quinine into the sciatic nerve by inadequately trained HCPs[20], which necessitated Uganda's policy change of the quinine injection site from the gluteus muscle to the thigh[21]. Adult doses of oral and intravenous quinine can be administered safely in pregnancy[15] but high doses may cause miscarriage[22].

Rare ADRs occur in [1-10] per 10,000 individuals $(0.01 \%$ to $0.1 \%)$ and very rare ADRs occur in fewer than 1 in $10,000(<0.01 \%)$ individuals receiving a specified medicine[14]. Twothirds (65\%, 95\% CI: 33/51) of rare suspected ADRs were serious thus underlining the importance of pharmacovigilance in tracking medication safety since reported rare ADRs are usually serious (incapacitating or life-threatening)[17]. Stevens-Johnson syndrome (SIS), a rare but serious skin-associated $\mathrm{ADR}$, was the most frequently reported, and it was mostly linked to the use of the antimicrobials (antibacterials and antiretrovirals), specifically nevirapine and 
cotrimoxazole. Similarly, antimicrobials were the most likely cause of SJS in a multicentre retrospective study conducted in India where nevirapine [9 of 32 cases] and cotrimoxazole [ 7 of 32 cases] also contributed most to the frequency of observed SJS[23]. The one case of diclofenac-associated haemoptysis described in this survey corroborates the findings of two recently documented case reports: one in Switzerland[24] and another in Belgium[25]. Rare and potentially fatal suspected ADRs are more likely to be identified after drugs have been licensed for use on the open market and voluntary ADR reporting by HCPs is a cornerstone of generating alerting adverse reaction signals[17].

One limitation to this study is that some suspected $\mathrm{ADR}(\mathrm{s})$ were described by more than one $\mathrm{HCP}(\mathrm{s})[5]$. Due to differences in the level of detail provided in the various ADR descriptions, we were unable to establish the full extent of multiply described incidents, a challenge that may be encountered by any voluntary ADR reporting system but one that can be mitigated if ADRs are described comprehensively. A second limitation is recall bias owing to the use of self-report but we mitigated this challenge by restricting the recall period for the ADR description to the previous one month. A third limitation is that neither the reporters nor the lead author performed ADR causality assessment to confirm causal relationships between the suspected ADRs and the implicated drugs. Although the rare, serious or unanticipated ADRs reported in this paper should be interpreted cautiously, the study investigators cross-checked each suspected ADR against authoritative reference texts using the online British National Formulary as the main reference[15]. We also maintain that HCPs need only to suspect and subsequently to report suspected adverse reactions to pharmacovigilance (PV) units without the prior requirement for formal causality assessment. Thus, NPCs should have the skilled personnel to be able to perform ADR causality assessment. Moreover, it is in this voluntary system of $\mathrm{ADR}$ reporting that a number of ADRs relating to post-marketed drugs are first identified.

Our study has generated key positive insights into how comprehensive and how alerting are the suspected ADRs reported by surveyed Ugandan HCPs.

\section{Conclusions}

Surveyed Ugandan HCPs who had suspected at least one ADR in the previous month competently provided comprehensive ADR descriptions: more, indeed, than are received per annum nationally. Properly analyzed, and with local feed-back, voluntary ADR reports by HCPs could be an essential alerting tool for identifying rare and serious suspected ADRs in Uganda.

\section{Supporting Information}

S1 Appendix. Ugandan suspected ADR form and reporting scheme. (PDF)

\section{S2 Appendix. Completeness quantification of ICSRs}

S3 Appendix. Study Questionnaire

$(\mathrm{PDF})$

\section{S4 Appendix. List of 241 ADR Descriptions}

(PDF)

S5 Appendix. Comprehensiveness of 241 ADR Descriptions

(PDF)

S6 Appendix. List of 106 serious ADR Descriptions

(PDF) 


\section{S7 Appendix. List of 59 severe ADR Descriptions}

(PDF)

S8 Appendix. Case reports of suspected ADRs

(PDF)

\section{Acknowledgments}

The authors wish to thank all the HCPs who agreed to participate in this study.

\section{Author Contributions}

Conceived and designed the experiments: RK SMB. Performed the experiments: RK. Analyzed the data: RK SMB. Contributed reagents/materials/analysis tools: RK SMB CK PW. Wrote the paper: RK SMB CK PW HBN.

\section{References}

1. Patel KJ, Kedia MS, Bajpai D, Mehta SS, Kshirsagar NA, Gogtay NJ. Evaluation of the prevalence and economic burden of adverse drug reactions presenting to the medical emergency department of a tertiary referral centre: a prospective study. BMC Clinical Pharmacology. 2007; 7:8. PMID: 17662147. Pubmed Central PMCID: PMC1963321. Epub 2007/07/31. eng.

2. Davies EC, Green CF, Taylor S, Williamson PR, Mottram DR, Pirmohamed M. Adverse drug reactions in hospital in-patients: a prospective analysis of 3695 patient-episodes. PLoS One. 2009; 4(2):e4439. PMID: 19209224. Epub 2009/02/12. eng. doi: 10.1371/journal.pone.0004439

3. Tumwikirize WA, Ogwal-Okeng JW, Vernby A, Anokbonggo WW, Gustafsson LL, Lundborg SC. Adverse drug reactions in patients admitted on internal medicine wards in a district and regional hospital in Uganda. Afr Health Sci. 2011 Mar; 11(1):72-8. PMID: 21572860. Epub 2011/05/17. eng.

4. WHO Draft Guidelines for Adverse Event Reporting and Learning Systems, (2005).

5. Kiguba R, Karamagi C, Waako P, Ndagije HB, Bird SM. Recognition and reporting of suspected adverse drug reactions by surveyed healthcare professionals in Uganda: key determinants. BMJ Open. 2014; 4(11):e005869. PMID: 25421337. doi: 10.1136/bmjopen-2014-005869

6. Pradel Editions. Adverse drug reactions: a practical guide to diagnosis and management. Baffins Lane, Chichester, West Sussex PO19 1UD, England: John Wiley and Sons Ltd; 1994 [cited 201419 September]. Available: http://eu.wiley.com/WileyCDA/WileyTitle/productCd-0471942111.html\#.

7. Hazell L, Shakir SA. Under-reporting of adverse drug reactions: a systematic review. Drug Safety. 2006; 29(5):385-96. PMID: 16689555. Epub 2006/05/13. eng.

8. National Pharmacovigilance Centre. Working together with the Public Health Programmes: a regulator's perspective for addressing the minimum requirements for Pharmacovigilance Kampala, Uganda: National Drug Authority; 2010 [cited 201321 June]. Available: http://www.who.int/medicines/areas/ quality_safety/regulation_legislation/icdra/WG-2_2Dec.pdf.

9. World Health Organization (WHO)—Uppsala Monitoring Centre (UMC). Documentation grading —how complete are the reports?: The Uppsala Monitoring Centre; 2011 [cited 20141 October]. Available: http://www.monitoringmedicines.org/graphics/26755.pdf.

10. World Health Organization (WHO) Uppsala Monitoring Centre (UMC). Causality Assessment of Suspected Adverse Reactions [20 September 2011]. Available: http://who-umc.org/Graphics/24734.pdf.

11. Gavaza P, Brown CM, Lawson KA, Rascati KL, Wilson JP, Steinhardt M. Influence of attitudes on pharmacists' intention to report serious adverse drug events to the Food and Drug Administration. $\mathrm{Br} \mathrm{J}$ Clin Pharmacol. 2011 Jul; 72(1):143-52. PMID: 21332572. Epub 2011/02/22. eng. doi: 10.1111/j.13652125.2011.03944.x

12. World Health Organization Collaborating Centre for International Drug Monitoring (UMC). Safety Monitoring of Medicinal Products: Guidelines for Setting Up and Running a Pharmacovigilance Centre 2000 [cited 201515 January]. Available: http://apps.who.int/medicinedocs/en/p/printable.html.

13. Division of AIDS. Table for Grading the Severity of Adult and Pediatric Adverse Events 2009 [cited 201431 July]. Available: http://rsc.tech-res.com/Document/safetyandpharmacovigilance/Table_for_ Grading_Severity_of_Adult_Pediatric_Adverse_Events.pdf. 
14. World Health Organization (WHO) Uppsala Monitoring Centre (UMC). Definitions [cited 20144 August]. Available: http://www.who.int/medicines/areas/quality_safety/safety_efficacy/trainingcourses/ definitions.pdf.

15. British National Formulary. London: BMJ Group and Pharmaceutical Press; 2013.

16. StataCorp. Stata Statistical Software: Release 12. College Station, TX: StataCorp LP; 2011.

17. Council for International Organizations of Medical Sciences (CIOMS). Benefit-Risk Balance for Marketed Drugs: Evaluating Safety Signals. Geneva1998 [cited 20144 August]. Available: http://www. cioms.ch/publications/g4-benefit-risk.pdf.

18. Etminan M, Gill S, Fitzgerald M, Samii A. Challenges and opportunities for pharmacoepidemiology in drug-therapy decision making. J Clin Pharmacol. 2006 Jan; 46(1):6-9. PMID: 16397278.

19. Talisuna AO, Staedke SG, D'Alessandro U. Pharmacovigilance of antimalarial treatment in Africa: is it possible?. Malar J. 2006; 5:50. PMID: 16780575. Epub 2006/06/20. eng.

20. Ekure J. Gluteal Fibrosis. A report of 28 cases from Kumi Hospital, Uganda. East and Central African Journal of Surgery. 2006 April 2006; 12(1):144-7.

21. National Pharmacovigilance Centre. What is current practice of Pharmacovigilance in Uganda? Kampala, Uganda: National Drug Authority; 2012 [cited 201311 December]. Available: http://africapv2012. files.wordpress.com/2012/04/day-2_5_country-presentations-nras_h-ngadije-uganda-compatibilitymode.pdf.

22. Actavis UK Ltd. Summary of Product Characteristics-Quinine Sulphate Tablets BP $300 \mathrm{mg}$ Whiddon Valley, Barnstaple, Devon, EX32 8NS, UK: Datapharm; 2013 [updated 22 April; cited 201419 September]. Available: http://www.medicines.org.uk/emc/medicine/24467.

23. Barvaliya M, Sanmukhani J, Patel T, Paliwal N, Shah H, Tripathi C. Drug-induced Stevens-Johnson syndrome (SJS), toxic epidermal necrolysis (TEN), and SJS-TEN overlap: a multicentric retrospective study. Journal of Postgraduate Medicine. 2011 Apr-Jun; 57(2):115-9. PMID: 21654132. Epub 2011/06/ 10. eng. doi: 10.4103/0022-3859.81865

24. Yiannakopoulou EC. Hemoptysis under diclofenac and antiplatelet doses of aspirin. Pharmacology. 2011; 87(1-2):1-4. PMID: 21150234. Epub 2010/12/15. eng.

25. Van Renterghem D, Depuydt C. Hemoptysis and Pulmonary Edema in a Scuba Diver Using Diclofenac. Pharmacology. 2012; 89(1-2):103-4. 